\title{
Capacity-Based Connectivity of MIMO Fading Ad-Hoc Networks
}

\author{
Hamid Jafarkhani Homayoun Yousefi'zadeh Javad Kazemitabar \\ Department of EECS \\ University of California, Irvine \\ [hamidj, hyousefi, skazemit] @uci.edu
}

\begin{abstract}
We study the problem of connectivity in MIMO fading ad-hoc networks. Based on a probabilistic analysis of achievable capacity on individual links of a random topology, we introduce a novel connectivity metric for wireless ad-hoc networks. We assume a pair of nodes are connected if their bi-directional capacity is more than a given threshold. Our metric is more sophisticated compared to previously proposed metrics of connectivity as it captures the effects of time-varying fading channel, power, and multiple antennas. Our results show that employing mobile nodes with multiple antennas enhances the connectivity of fading wireless ad-hoc networks.
\end{abstract}

Index Terms- Wireless Ad-Hoc Networks, Fading Channel, Connectivity, MIMO, Capacity.

\section{INTRODUCTION}

Strong tendency for the deployment of ad-hoc networks has encountered major challenges due to sometimes conflicting time-varying fading channel, connectivity, capacity, and power issues. This paper offers a framework for studying the connectivity phenomenon in Multiple-Input Multiple-Output (MIMO) fading ad-hoc networks based on the achievable link capacity.

Investigating the connectivity of radio networks goes back to four decades ago. In his pioneering work, Gilbert [9] studied the connectivity of infinite random networks relying on the so-called geometric disk model. In the geometric disk model, a random topology network is represented by a disk graph in which two nodes are considered directly connected if their distance is smaller than a given transmission radius. The work of Gilbert showed that there exists a minimum number of nodes within a transmission range above which a random graph is almost surely connected. Recently, the connectivity subject has received much attention due to the deployment of wireless adhoc networks. Some of the recent follow-on studies about the connectivity of infinite random networks relying on the geometric disk model include the work of Booth et al. [4], Philips et al. [13], and Quintanilla et al. [14]. In addition, a survey of the literature reveals a large number of articles in the context of connectivity of ad-hoc networks with a finite number of mobile nodes. Some of the related articles in this area include the work of Cheng et al. [5], Santi et al. [16], Bettstetter [3], and Doussi et al. [6].

Although an attractive abstract model for studying the connectivity, the geometric disk model is far from the reality of wireless networks. The main disadvantages of the disk model have to do with not considering the effects of attenuation, interference, and noise. Gupta et al. [10] proposed the use of

This work was supported in part by the U. S. Army Research Office under the Multi-University Research Initiative (MURI) grant number W911NF-04-10224 . signal-to-interference-noise-ratio $(S I N R)$ as the metric of connectivity in wireless ad-hoc networks. According to $S I N R$ metric, two nodes in a random topology are directly connected if their minimum $S I N R$ is greater than a given threshold. Baccelli et al. [2] utilized $S I N R$ model under Poisson assumptions in the context of infinite CDMA networks. Relying on the same $S I N R$ connectivity metric, Dousse et al. [7] showed that if both node density per unit area and $S I N R$ are sufficiently high, the resulting infinite graph of an ad-hoc network is almost surely connected. Interestingly, connectivity in random networks represented by graphs of mixed short and long edges can also be related to small world networks [19].

While $S I N R$ is a more realistic metric of connectivity compared to the geometric disk model for wireless ad-hoc networks, it is still not fully capable of capturing the physical connectivity phenomenon. In reality, a pair of nodes are connected if a sequence of transmitted symbols from one can be received at another. In addition, the considerations of time and frequency can also affect the interpretation of connectivity.

The use of space-time coding techniques in wireless networks is of interest because they can substantially reduce the effects of multipath fading in the wireless channels through antenna diversity. Transmit antenna diversity in the form of spacetime block codes (STBCs) of [1] and [17] has been adopted in WCDMA and CDMA2000 standards. It is also being considered in many current wireless standard efforts. Receive antenna diversity schemes such as Maximum Ratio Combining (MRC) are already in widespread use in communication systems.

The contributions of our work is in the introduction of a probabilistic connectivity metric for wireless ad-hoc networks. We rely on an analysis of the time-varying fading wireless network to define a connectivity metric based on the capacity of MIMO channels.

The rest of this paper is organized as follows. Section II provides properties of random variables some that will be used in our analysis. Section III investigates the problem of connectivity based on the probabilistic concept of capacity in MIMO channels. In Section IV, we numerically validate our connectivity analysis results. Our findings show that utilizing spacetime coded mobile nodes can improve the connectivity of adhoc networks under our connectivity metric. Finally, Section V concludes this paper.

\section{Fundamental Properties of Random VARiables}

In this section, we provide a review of few fundamental properties of random variables. 
We list four fundamental properties of the Probability Density Functions (PDFs) of random variables as discussed by [12] and [15].

- Property 1: If $x$ and $y$ are random variables satisfying $y=$ $g(x)$, then the PDF of $y$ satisfies $f_{y}(y)=\frac{f_{x}\left(x_{1}\right)}{g^{\prime}\left(x_{1}\right)}+\cdots+$ $\frac{f_{x}\left(x_{q}\right)}{g^{\prime}\left(x_{q}\right)}$ where $x_{1}, \cdots, x_{q}$ are the real roots of $y=g(x)$ and $g^{\prime}(x)$ represents the derivative of $g(x)$.

- Property 2: If the PDF set $\left\{f_{x_{1}}\left(x_{1}\right), \cdots, f_{x_{q}}\left(x_{q}\right)\right\}$ is associated with the set of independent random variables $\left\{x_{1}, \cdots, x_{q}\right\}$, then the PDF of their sum $z=x_{1}+\cdots+x_{q}$ is calculated as $f_{z}(z)=f_{x_{1}}(z) * \cdots * f_{x_{q}}(z)$ where $*$ represents the convolution operator.

- Property 3: If $x, y$, and $z$ are random variables satisfying $z=x^{2}+y^{2}$, the PDF of the random variable $z$ is specified as $f_{z}(z)=\int_{-\sqrt{z}}^{\sqrt{z}} \frac{1}{\sqrt{z-y^{2}}}\left\{f_{x y}\left(\sqrt{z-y^{2}}, y\right)+\right.$ $\left.f_{x y}\left(-\sqrt{z-y^{2}}, y\right)\right\} d y$.

- Property 4: Given the individual PDFs of $k$ Identically and Independently Distributed (IID) exponential random variables $x_{1}, \cdots, x_{k}$, with the common parameter $\lambda$ the PDF of their sum $z=x_{1}+\cdots+x_{k}$ is expressed by a gamma distribution with parameters $\mathrm{k}$ and $\lambda$ as

$$
f_{z}(z)=\frac{z^{k-1}}{(k-1) !} \lambda^{k} e^{-\lambda z}, \quad z>0
$$

\section{A CAPACITy-BASEd CONneCtivity Metric}

The discussion of this section revolves around defining a probabilistic metric of connectivity that takes into consideration the details of time-varying wireless channel and the underlying communication system. Our probabilistic metric is defined based on an outage capacity analysis for MIMO channels.

Calculating estimates or upper bounds of the capacity in the case of uncorrelated and correlated Single-Input SingleOutput (SISO) and MIMO channels both with Gaussian and non-Gaussian noise has been the subject of heavy research in the past years. The concept of outage capacity was first introduced by Foschini et al. [8]. Outage capacity provides an elegant description of the achievable rate of a communication channel. Simply put, it represents a probabilistic measure of the maximum number of bits per cycle that can be transmitted for a given probability of error. Foschini et al. [8] also provided approximations of the capacity of IID MIMO Rayleigh channels. Telatar [18] provided a treatment of calculating the capacity of correlated MIMO channels with Gaussian noise. Kang et al. [11] numerically verified that the approximations of capacity derived in [8] work well under various fading conditions in the presence of Rayleigh distributed interference, for a wide range of $S I N R$, and even when the channel is semi-temporally correlated.

Our discussion below represents a treatment of the subject material relying on the cited literature articles above. The analysis is carried out by working with the input and output signals of a Rayleigh fading channel.

We consider an ad-hoc topology consisting of a number of links. Further, we assume a MIMO channel with $M_{i}$ transmit antennas and $N_{i}$ receive antennas is associated with link $i$ of the topology. Let us also assume that the $N_{i} \times M_{j}$ matrix $H_{i j}$ represents the Rayleigh fading channel between the transmitter of link $j$ and the receiver of link $i$. Denoting $\mathbf{S}_{i}$ as the $M_{i} \times 1$ signal vector transmitted from link $i$, the received signal vector at link $i$ is the following $N_{i} \times 1$ vector

$$
\mathbf{R}_{i}(t)=H_{i i}(t) \mathbf{S}_{i}(t)+\sum_{j \neq i} H_{i j}(t) \mathbf{S}_{j}(t)+\mathbf{n}_{i}(t)
$$

where the channel matrices $H_{i j}$ consist of complex Gaussian random variable elements. The above equation can be rewritten as

$$
\mathbf{R}_{i}(t)=H_{i i}(t) \mathbf{S}_{i}(t)+\mathbf{n}_{i}^{\prime}(t)
$$

where $\mathbf{n}_{i}^{\prime}(t)=\sum_{j \neq i} H_{i j}(t) \mathbf{S}_{j}(t)+\mathbf{n}_{i}(t)$. We assume that the receiver of link $i$ knows the channel matrix $H_{i i}$ while the transmitter of link $i$ only knows its distribution. The quantities $\left(\mathbf{n}_{i}^{\prime} \mid H_{i j}\right)$ can be considered to form a Gaussian random process due to the following lines of reasoning. We know that the codewords $\mathbf{S}_{j}$ should be chosen from a Gaussian distribution to be capacity achieving. Further, $H_{i j}$ 's are known at the receiver. Since the elements $H_{i j} \mathbf{S}_{j}$ are linear combinations of some independent Gaussian random variables, they are themselves Gaussian. In addition, any $\mathbf{S}_{j}$ or $\mathbf{n}_{i}$ term at time slot $t$ is independent from its counter part at other time slots. The latter is due to the fact that transmitter assigns the codewords independently at each time slot as the result of not knowing the channel. Therefore, $\left(\mathbf{n}_{i}^{\prime} \mid H_{i j}\right)$ is a Gaussian random process. The covariance matrix for this resulting noise term is

$$
\begin{aligned}
K & =E\left\{\mathbf{n}_{i}^{\prime} \cdot \mathbf{n}_{i}^{\prime \dagger}\right\} \\
& =E\left\{\left(\sum_{j \neq i} H_{i j} \mathbf{S}_{j}+\mathbf{n}_{i}\right) \cdot\left(\sum_{k \neq i} H_{i k} \mathbf{S}_{k}+\mathbf{n}_{i}\right)^{\dagger}\right\} \\
& =E\left\{\sum_{j \neq i} H_{i j} \mathbf{S}_{j} \mathbf{S}_{j}^{\dagger} H_{i j}^{\dagger}\right\}+\sigma_{n}^{2} I
\end{aligned}
$$

where, the superscript $\dagger$ indicates the Hermitian operator. Since we are assuming that $H_{i j}$ coefficients are known at the receiver,

$$
K=\sum_{j \neq i} H_{i j} E\left\{\mathbf{S}_{j} \mathbf{S}_{j}^{\dagger}\right\} H_{i j}^{\dagger}+\sigma_{n}^{2} I=\sum_{j \neq i} H_{i j} \Phi_{j} H_{i j}^{\dagger}+\sigma_{n}^{2} I
$$

where $I$ is the identity matrix and $\Phi_{j}$ indicates the covariance matrix of the code used in link $j$. Then, the mutual information $\mathcal{I}$ between $\mathbf{S}_{i}$ and $\mathbf{R}_{i}$ is derived as ${ }^{1}$

$$
\mathcal{I}\left(\mathbf{S}_{i} ; \mathbf{R}_{i}\right)=\log _{2} \operatorname{det}\left(I+K^{-1} H_{i i} \Phi_{i} H_{i i}^{\dagger}\right)
$$

To find the capacity, one needs to maximize $\mathcal{I}\left(\mathbf{S}_{i} ; \mathbf{R}_{i}\right)$ subject to a transmission power constraint $\operatorname{Tr}\left(\Phi_{i}\right) \leq P_{i}$ on link $i$ where $\operatorname{Tr}\left(\Phi_{i}\right)$ and $P_{i}$ denote the trace of $\Phi_{i}$ and the transmission power of link $i$, respectively. Since the receiver knows the channel, only some statistics are needed to determine the capacity.

The choice of covariance matrix achieving the capacity in Equation (6) depends on the realization of the channel matrix. When the channel is not known at the transmitter, the best strategy is to distribute the input power equally among the transmit antennas. The latter results in a covariance matrix $\Phi_{i}$ that is

\footnotetext{
${ }^{1}$ The symbol $\mathcal{I}$ used to denote mutual information should be distinguished from the symbol $I$ to denote the identity matrix.
} 
a multiple of the identity matrix. Considering the constraint $\operatorname{Tr}\left(\Phi_{i}\right) \leq P_{i}$, we have $\Phi_{i}=\frac{P_{i}}{M_{i}} I$ resulting in the following capacity determination:

$$
C_{i}=\log _{2} \operatorname{det}\left(I+\frac{P_{i}}{M_{i}} K^{-1} H_{i i} H_{i i}^{\dagger}\right) \quad \text { bits } / \mathrm{sec} / H z
$$

In the most general case, the capacity expression of Equation (7) can be only calculated numerically. For a special case in which $K$ is a multiple of the identity matrix, we can offer analytical results. As the central limit theorem suggests, the latter is not a bad assumption. Using Equation (5) and assuming a large number of interfering nodes, one can consider $K$ to be a multiple of the identity matrix. As such, it can be expressed as

$$
K \simeq\left[\bar{P}_{i}^{(I)}+\bar{P}_{i}^{(n)}\right] I
$$

Therefore, Equation (7) can be rewritten as follows

$$
C_{i}=\log _{2} \operatorname{det}\left(I+\frac{\overline{S I N R}_{i}}{M_{i}} H_{i i} H_{i i}^{\dagger}\right) \quad \text { bits } / \text { sec } / H z
$$

with $\overline{S I N R}_{i}$ denoting the average signal-to-interference-noiseratio. Next, we note that the capacity in Equation (9) is defined for a fixed realization of the fading channel $H_{i i}$ at link $i$ over a large block length. Every realization of the channel has some probability attached to it through the statistical model of $H_{i i}$. We assume that the matrix $H_{i i}$ consists of zero-mean Gaussian random variables, i.e., each element of the matrix has a fading envelope described by Rayleigh distribution [12]. As a direct result of Property 2, we know that the sum of $q$ zero-mean IID complex Gaussian random variables with a standard deviation $\frac{1}{\sqrt{2 \lambda}}$ is a zero-mean Gaussian random variable with a standard deviation $\sqrt{\frac{q}{2 \lambda}}$. Since the channel matrices $H_{i i}$ are random in nature, the capacity in Equation (9) can be considered as a random variable.

According to Singular Value Decomposition (SVD) theorem, $C_{i}$ can be calculated in terms of the positive eigenvalues of $H_{i i} H_{i i}^{\dagger}$ as

$$
C_{i}=\sum_{l=1}^{\rho} \log _{2}\left[1+\frac{\overline{S I N R}_{i}}{M_{i}} \sigma_{l}\right] \quad \text { bits } / \mathrm{sec} / \mathrm{Hz}
$$

where $\sigma_{l}$ 's with $l \in\{1, \cdots, \rho\}$ denote the positive eigenvalues of $H_{i i} H_{i i}^{\dagger}$ and $\rho$ is the rank of $H_{i i}$. Therefore, the capacity $C_{i}$ represents a scalar function of the set of random variables $\left\{\sigma_{1}, \cdots, \sigma_{\rho}\right\}$. The PDF of capacity can be calculated as described below and depending on the values of $M_{i}$ and $N_{i}$.

For the case of $M_{i}=N_{i}=1$, we have $H_{i i}=x+j y$ with $x$ and $y$ representing zero-mean IID Gaussian random variables and $H_{i i} H_{i i}^{\dagger}=x^{2}+y^{2}$. We utilize Property 3 for the pair of zero-mean Gaussian random variables $x$ and $y$ with a standard deviation of $\sqrt{2 \lambda}$ to conclude that the PDF of the scalar $H_{i i} H_{i i}^{\dagger}$ is described in the form of

$$
f_{z}(z)=\lambda e^{-\lambda z}
$$

The PDF identified above represents the only positive eigenvalue of the scalar function $H_{i i} H_{i i}^{\dagger}$. For the case of $M_{i}=2$ and $N_{i}=1$,

$$
\begin{aligned}
H_{i i} H_{i i}^{\dagger} & =\left[\begin{array}{ll}
x_{1}+j y_{1} & x_{2}+j y_{2}
\end{array}\right]\left[\begin{array}{l}
x_{1}-j y_{1} \\
x_{2}-j y_{2}
\end{array}\right] \\
& =\left(x_{1}^{2}+y_{1}^{2}\right)+\left(x_{2}^{2}+y_{2}^{2}\right)
\end{aligned}
$$

where $x_{1}, y_{1}, x_{2}$, and $y_{2}$ represent zero-mean IID Gaussian random variables. Defining $z_{1}=x_{1}^{2}+y_{1}^{2}$ and $z_{2}=x_{2}^{2}+y_{2}^{2}$, the PDF of the scalar function $H_{i i} H_{i i}^{\dagger}$ can be derived from Property 4 as

$$
f_{z}(z)=\lambda^{2} z e^{-\lambda^{2} z}, \quad z>0
$$

Again, the PDF identified above represents the only positive eigenvalue of $H_{i i} H_{i i}^{\dagger}$. For the case of $M_{i}=1$ and $N_{i}=2$,

$$
\begin{aligned}
& H_{i i} H_{i i}^{\dagger}=\left[\begin{array}{l}
x_{1}+j y_{1} \\
x_{2}+j y_{2}
\end{array}\right]\left[\begin{array}{ll}
x_{1}-j y_{1} & x_{2}-j y_{2}
\end{array}\right] \\
& =\left[\begin{array}{ll}
\left(x_{1}^{2}+y_{1}^{2}\right) & x_{1} x_{2}+y_{1} y_{2}+j\left(x_{1} y_{2}-x_{2} y_{1}\right) \\
x_{1} x_{2}+y_{1} y_{2}-j\left(x_{1} y_{2}-x_{2} y_{1}\right) & \left.x_{2}^{2}+y_{2}^{2}\right)
\end{array}\right]
\end{aligned}
$$

where $x_{1}, y_{1}, x_{2}$, and $y_{2}$ represent zero-mean IID Gaussian random variables. Calculating the eigenvalues of $H_{i i} H_{i i}^{\dagger}$ yields a zero eigenvalue and a positive eigenvalue of $\left(x_{1}^{2}+y_{1}^{2}\right)+\left(x_{2}^{2}+\right.$ $\left.y_{2}^{2}\right)$. The PDF of the random variable associated with the positive eigenvalue is the same as the one identified by Equation (13). The results for the case of $M_{i}=2$ and $N_{i}=2$ are calculated similar to the case of $M_{i}=2$ and $N_{i}=1$ with an $H_{i i}$ matrix consisting of four pairs of complex Gaussian random variables.

Treating capacity as a random variable with a given PDF provides us with an opportunity to represent a novel connectivity metric based on the concept of outage capacity. We introduce our connectivity metric as

$$
\operatorname{Pr}\left(C_{i}<C_{\text {out }}\right)<P_{\text {out }}
$$

where $\operatorname{Pr}(),. C_{\text {out }}$, and $P_{\text {out }}$ represent probability, the threshold of connectivity also known as outage capacity, and the outage probability, respectively. It differs slightly from that of [18]. According to [18], the outage is defined as

$$
\inf _{\operatorname{Tr}\left(Q_{i}\right) \leq M_{i}} \operatorname{Pr}\left(C_{i}<C_{\text {out }}\right)<P_{\text {out }}
$$

The main difference between the two definitions is that the latter may assign zero power to some of the transmit antennas while the former utilizes all of the antennas. According to our outage capacity metric matching the former definition, two nodes are connected if the probability of their outage capacity is less than a given outage probability.

We remind that the advantage of using the connectivity metric of this section compared to an $S I N R$ metric is that the metric of this section can capture the fading characteristics of the wireless channel as well as the effects of utilizing multiple antennas in the nodes, through $H_{i i} H_{i i}^{\dagger}$ term.

\section{Connectivity Experiments}

In this section, we apply our proposed connectivity scheme to a moderate size random ad-hoc topology. In order to provide a meaningful basis of comparison, we compare our results for 
the same random topology. In our random topology, 200 nodes are distributed on a 2-D domain with an area of 1000 square meters according to a Poisson point process.

Before proceeding with the explanation of our numerical results, we note that we are investigating the connectivity of wireless ad-hoc networks accommodating mobile nodes with both single and double transmit antennas. In the case of a double transmit antenna mobile node, we assume that two signals are transmitted simultaneously from the two transmit antennas at each time slot. In addition, we assume that the slow fading wireless channel characterized by a Rayleigh distribution is quasi-static and flat implying that the path gains are constant over a frame but vary independently from one frame to another.

The following describes general settings of our experiments. We assume that each node utilizes a total transmission power of $P=1 W$ on the combined set of its outgoing links. In the case of multiple antenna nodes, the total transmission power is split equally among the antenna paths. The expected value of the noise power on each path is assumed to be $10 \mu \mathrm{W}$. Depending on a specific experiment, a pair of nodes are considered to be immediate neighbors and form a direct link $L_{i}$ if the probabilistic connectivity metric of (15) holds. We note that link connectivity may be directional implying that a first node can transmit to a second node while the second node may not be able to transmit to the first node. In our experiments, we consider link connectivity exists only if both nodes can transmit and receive from each other under a connectivity criterion.

For the random topology described above, we consider three scenarios. In the first scenario to which we refer as the $1 \times 1$ case, the network is only accommodating single antenna mobile nodes. In the second scenario to which we refer as the HYBRID case, half of the nodes are randomly selected to be equipped with double antennas while the other half are equipped with single antennas. In the third scenario to which we refer as the $2 \times 2$ case, the network is only accommodating double antenna mobile nodes.

We provide the results of our experiments in the case of probabilistic measure of (15). The illustrations of Fig. 1 show the connectivity graphs of our connectivity metric for the given random topology network. Reviewing the connectivity graphs, we observe that the connectivity graphs of the topology vary depending on not only the signal-to-interference-noise-ratio but the capacity. Hence, a pure measurement of the signal strength such as $S I R, S N R$, or $S I N R$ is not quite capable of describing the connectivity phenomenon. On the contrary, utilizing our proposed metric provides a better way of properly capturing the effects of the quantities of interest when investigating connectivity. A significant observation is that to our expectation equipping mobile nodes with a higher number of antennas improves the connectivity results. From the results of the experiments, we can also calculate the percentages of the nodes belonging to the largest connected cluster of nodes. Utilizing the connectivity metric of (15), Table I reports the connectivity results for three different combination of choices of $C_{\text {out }}$ and $P_{\text {out }}$ with similar other settings. We observe that decreasing the value of $C_{\text {out }}$ and increasing the value of $P_{\text {out }}$ increases the size of the largest cluster of the connectivity graph.

We note that the connectivity of an ad-hoc network depends
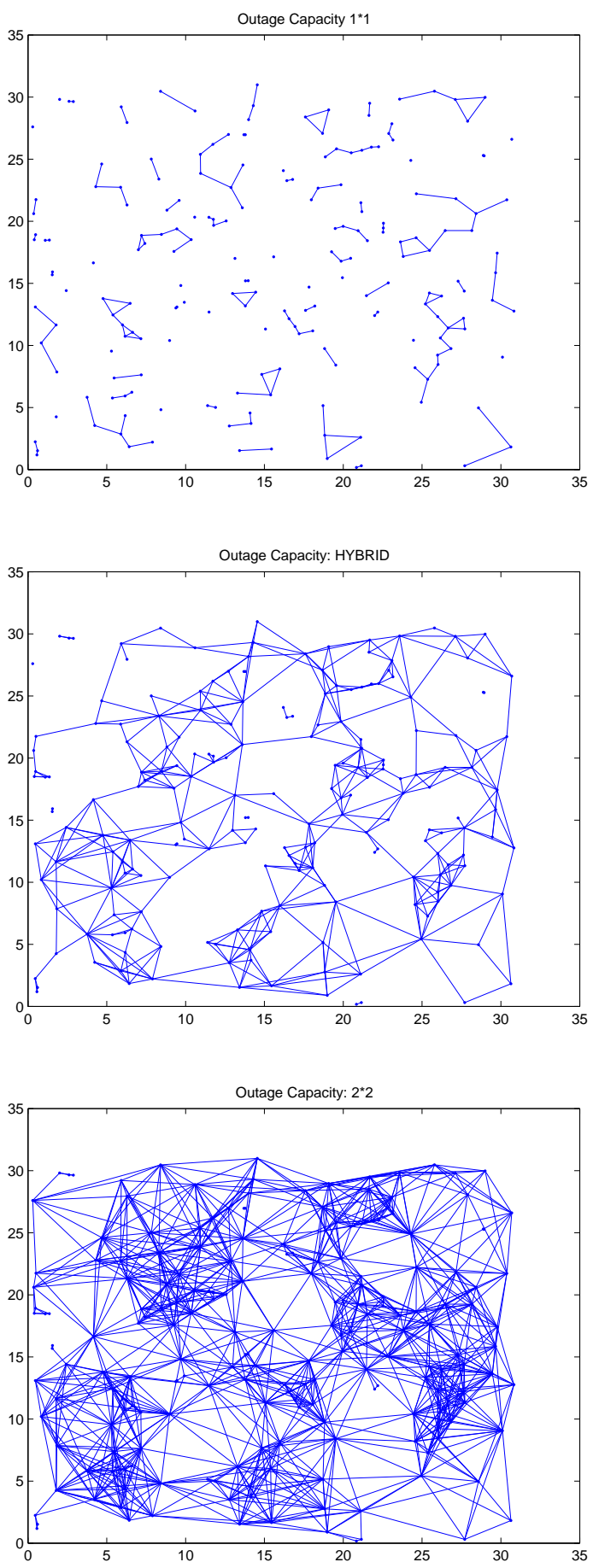

Fig. 1. Connectivity graphs of a random topology with connectivity thresholds of $C_{\text {out }}=1 \mathrm{bit} / \mathrm{sec} / \mathrm{Hz}$ and $P_{\text {out }}=0.02$. The figures from the top to the bottom correspond to single antenna, hybrid, and double antenna mobile nodes.

TABLE I

A COMPARISON OF THE RELATIVE SIZES OF THE LARGEST CONNECTED CLUSTER UTILIZING OUTAGE CAPACITY CONNECTIVITY METRIC.

\begin{tabular}{|c||c|c|c|}
\hline & $\begin{array}{c}C_{\text {out }}=2 \\
P_{\text {out }}=0.01\end{array}$ & $\begin{array}{c}C_{\text {out }}=1.5 \\
P_{\text {out }}=0.01\end{array}$ & $\begin{array}{c}C_{\text {out }}=1 \\
P_{\text {out }}=0.02\end{array}$ \\
\hline $1 \times 1$ & $1.5 \%$ & $2 \%$ & $7 \%$ \\
\hline HYBRID & $12 \%$ & $31.5 \%$ & $90.5 \%$ \\
\hline $2 \times 2$ & $90.5 \%$ & $94 \%$ & $98 \%$ \\
\hline
\end{tabular}


on the choice of connectivity parameters and the specific topology of the network. Additionally based on our results, we anticipate that equipping more mobile nodes with multiple antennas improves the connectivity of an ad-hoc network for any given topology and set of parameters.

\section{CONCLUSION}

We studied the problem of connectivity in fading wireless ad-hoc networks. We defined a probabilistic metric of connectivity based on the capacity of MIMO channels. We argued that our connectivity metric is more sophisticated than the previously proposed connectivity metrics such as signal strength due to the fact that it captures the time-varying fading, transmission power, and multiple antenna characteristics of wireless nodes. Our results showed that the use of multiple antenna mobile nodes improves the connectivity of ad-hoc networks.

\section{ACKNOWLEDGMENT}

The authors would like to thank Syed A. Jafar for useful discussions about the capacity of MIMO channels.

\section{REFERENCES}

[1] S.M. Alamouti, "A Simple Transmitter Diversity Scheme for Wireless Communications," IEEE JSAC, November 1998.

[2] F. Baccelli, B. Blaszczyszyn, "On A Coverage Process Ranging from the Boolean Model to the Poisson Voronoi Tessellation, with Applications to Wireless Communications," Advance Applied Probability, vol. 33(2), 2001.

[3] C. Bettstetter, "On the Minimum Node Degree and Connectivity of a Wireless Multihop Network," In Proc. ACM MOBIHOC, 2002.

[4] L. Booth, J. Bruck, M. Franschetti, R. Meester, "Continuum Percolation and the Geometry of Wireless Networks," Annals of Applied Probability, 2002.

[5] Y.-C. Cheng, T. G. Robertazzi, "Critical Connectivity Phenomena in Multihop Radio Models,” IEEE Trans. Communications, July 1989.

[6] O. Dousse, P. Thiran, M. Hasler, "Connectivity in Ad-Hoc and Hybrid Networks," In Proc. IEEE INFOCOM, 2002.

[7] O. Dousse, F. Baccelli, P. Thiran, "Impact of Interferences on Connectivity in Ad-Hoc and Networks," In Proc. IEEE INFOCOM, 2003.

[8] G.J. Foschini, M.J. Gans, "On Limits of Wireless Communication in a Fading Environment When Using Multiple Antennas," Wireless Personal Communications, March 1998.

[9] E. N. Gilbert, "Random Plane Networks," SIAM J., vol. 9, pp. 533-543, 1961.

[10] P. Gupta, P. R. Kumar, “The Capacity of Wireless Networks,” IEEE Trans. Information Theory, March 2000.

[11] M. Kang, M.-S. Alouini, G.E. Oien, "How Accurate Are the Gaussian and Gamma Approximations to the Outage Capacity of MIMO channels?," In Proc. Baiona Workshop on Signal Processing in Communications, 2003.

[12] A. Papoulis, S.U. Pillai, "Probability, Random Variables, and Stochastic Processes, Fourth Edition,” McGraw-Hill, ISBN 0071122567, 2002.

[13] T.K. Philips, S.S. Panwar, A.N. Tantawi, "Connectivity Properties of a Packet Radio Network Model," IEEE Trans. Information Theory, September 1989.

[14] S. Quintanilla, S. Torquato, R.M. Ziff, "Efficient Measurements of the Percolation Threshold for the Fully Penetrable Disks," Journal of Physics, October 2000.

[15] S. Ross, "A First Course in Probability, Sixth Edition" Prentice-Hall, ISBN 0130338516, 2001.

[16] P. Santi, D.M. Blough, "An Evaluation of Connectivity in Mobile Wireless Ad Hoc Networks," In Proc. IEEE DSN, 2002.

[17] V. Tarokh, H. Jafarkhani, A.R. Calderbank, "Space-Time Block Coding from Orthogonal Designs," IEEE Trans. Information Theory, July 1999.

[18] I.E. Telatar, "Capacity of Multi-Antenna Gaussian Channels," European Trans. Telecommunications, November-December 1999.

[19] D. Watts, S. Strogatz, "Collective Dynamics of Small-World Networks," Nature, June 1998. 